\title{
BASES DE LA EPISTEMOLOGÍA A COMIENZOS DEL SIGLO XXI
}

\author{
FOUNDATIONS OF EPISTEMOLOGY IN EARLY XXI CENTURY \\ Miguel Martínez Miguélez $Z^{1}$ \\ Universidad Simón Bolívar de Caracas, Venezuela \\ (RECIBIDO EL 15/01/2010, ACEPTADO EL 20/06/2010)
}

\begin{abstract}
RESUMEN
Las realidades del mundo actual, tanto en su aspecto personal y familiar, como en el social, político, religioso y empresarial, han adquirido, en el siglo XX, un alto nivel de complejidad. Esta situación exige, para su estudio y comprensión, un enfoque de investigación transdisciplinario, integrado y sistémico, el cual, a su vez, necesita unas bases epistemológicas acordes con su propia y especial naturaleza, ya que vivimos una transformación radical del concepto de conocimiento y del concepto de ciencia, es decir, del criterio de racionalidad científica. Será objetivo fundamental de este estudio clarificar e ilustrar que el problema reside en el concepto restrictivo de "cientificidad" adoptado, especialmente en las ciencias humanas, que mutila la legitimidad y derecho a existir de una gran riqueza de la dotación más típicamente humana, como los procesos que se asientan en el uso de la libertad y de la creatividad. Igualmente haremos énfasis en la necesidad de una revisión de los marcos conceptuales, teorías y métodos de la filosofía de la ciencia involucrada y tratar de aclarar los fundamentos y naturaleza de los procesos mentales psicológicos que están implícitos en ella.
\end{abstract}

Palabras clave: Epistemología; racionalidad; sistémico; transdisciplinaridad.

\begin{abstract}
The realities of today's world, both in its personal and family level, as in the social, political, religious and business one, have acquired, in the twentieth century, a high level of complexity. This calls, for its study and understanding, an interdisciplinary research approach, integrated and systemic, which, in turn, needs a consistent epistemological bases with its own special nature, because we live a radical transformation of the concept of knowledge and the concept of science, i.e., the criterion of scientific rationality. It will be fundamental objective of this study to clarify and illustrate that the problem lies in the restrictive concept of "scientificity" adopted, especially in the humanities, which cripples the legitimacy and right to exist of a wealth of more typical human endowment, as processes that are based on the use of freedom and creativity. Also, we will emphasize the need for a review of conceptual frameworks, theories and methods of philosophy of science involved, and seek to clarify the rationale and nature of the psychological mental processes that are implicit in it.
\end{abstract}

Keywords: Epistemology, rationality, systemic, transdisciplinarity.

1 El Dr. Miguel Martínez M. es profesor titular de la Universidad Simón Bolívar de Caracas (Venezuela), y responsable de la línea de investigación "Epistemología y metodología cualitativa".

E-mail: miguelm@usb.ve - Web: http://prof.usb.ve/miguelm; http://miguelmartinezm.atspace.com 


\section{INTRODUCCIÓN}

Las realidades del mundo actual han ido adquiriendo un nivel de complejidad creciente cada vez más intrincado y enrevesado. Esto está sucediendo en todas las áreas de la vida, tanto al nivel personal y familiar, como en el social, empresarial, político y religioso. El número de factores que entran en acción y las múltiples relaciones que crean entre sí exigen, para su estudio y comprensión, un enfoque transdisciplinario, integrado y sistémico, ya que no se trata solo de un agregado de elementos sino de componentes constituyentes que forman un sistema y crean un nuevo orden.

Será objetivo fundamental de este estudio tratar de aclarar el contenido de los términos involucrados, siguiendo no solo su etimología y la epistemología en que son usados, sino, y sobre todo, la naturaleza de los procesos mentales psicológicos que están implícitos en ellos. Igualmente seguiremos la claridad del "modelo médico": exponer, en la primera parte, lo relacionado con el diagnóstico de la situación actual y, después, en la segunda, aquellas sugerencias y propuestas que pudieran constituir una terapia epistemológica para tal situación.

Durante los últimos 20 años, la Unesco, como Organización de las Naciones Unidas para la educación, la ciencia y la cultura, viene insistiendo y nos viene alertando sobre una serie de ideas de máxima relevancia (Ciret-Unesco, 1997, 2000; Unesco, 1998). Entre esas ideas están las siguientes afirmaciones:

-_ Los países en desarrollo solo lo alcanzarán con una calificada y competente preparación de sus profesionales.

-_ La desorientación de la universidad es un fenómeno mundial.

-_ Los cambios mundiales tienen un ritmo acelerado.

-- La lógica positivista y pensamiento único generan pobreza.

-_ No podemos seguir parcelando el saber; necesitamos un enfoque transdisciplinario.

-- Es urgente una visión transnacional, transcultural, transpolítica y transreligiosa.

-_ Debemos adoptar un paradigma sistémico para entender la complejidad.

-_ Es necesario rehacer los planes de estudio.

-_ El diálogo como método es imprescindible.

Todo esto nos encamina hacia una seria reflexión sobre los fundamentos de la ciencia, a realizar una revisión de sus bases y de sus marcos conceptuales, teorías y métodos.

Mc-Luhan solía decir: "yo no sé quién descubrió el agua por primera vez, pero estoy seguro que no fueron los peces”. En efecto, los peces, rodeados de agua por todas partes, no la pueden ver. Tampoco nosotros podemos descubrir una realidad que damos, ingenuamente, por supuesta. Pero tenemos algo que no tienen los peces: el poder de la reflexión, que puede analizarse a sí misma.

Precisamente, I. Kant (1973) nos invita a realizar esa tarea, cuando, en la introducción de su obra máxima, La crítica de la razón pura, nos dice: “el maduro juicio de nuestra época 
no quiere seguir contentándose con un saber aparente y exige de la razón la más difícil de sus tareas, a saber: que de nuevo emprenda su propio conocimiento” (p. 121).

Aunque esa tarea ha sido siempre la principal de la filosofía, en nuestros tiempos comenzó, en forma amplia, continua y consistente, a mediados del siglo XX. Efectivamente, hasta la década de los años 50 - salvo contadas excepciones como la de los físicos de la teoría cuántica, la de los psicólogos de la Gestalt y la de la Teoría de sistemas- el principio básico de la ciencia era el principio de reducción, el cual hacía consistir el conocimiento del todo en la percepción de sus partes, partes que consideraba aisladamente.

Hoy, en cambio, sabemos que no podemos buscarle soluciones únicamente económicas a los problemas económicos, ni soluciones únicamente políticas a los problemas políticos, ni soluciones únicamente sociales a los problemas sociales, ya que —como dice Whitehead-, "quien conoce solamente su propia disciplina ni siquiera esa disciplina conoce". En todos los campos se constata que la mayoría de los problemas no pueden resolverse al nivel en que vienen planteados, que su naturaleza forma como un rizoma complejo de muy variadas interacciones.

Por todo ello, la tarea a realizar no es fácil ya que, si hay algo verdaderamente difícil, es la toma de conciencia crítica de nuestros propios presupuestos, de nuestro propio punto de vista, pues frecuentemente están arraigados en un apego afectivo, en un acto de fe gratuito e inconsciente. Por esto, Kant, muy consciente de ello, recomendaba a sus alumnos que miraran no tanto a lo que la gente decía que veía, sino que miraran y examinaran el ojo de esas personas. Y el gran físico cuántico Werner Heisenberg señalaba que "nunca observamos la naturaleza de las cosas en sí mismas, sino esa naturaleza expuesta a nuestro método de investigación" (1958: 58). Este mismo físico revela que una vez Einstein le dijo: "el hecho de que usted pueda observar una cosa o no, depende de la teoría que usted use. Es la teoría la que decide lo que puede ser observado" (en Bronowski, 1979: 249).

\section{HACIA UN NUEVO ENFOQUE DE LA CIENCIA}

\section{Necesidad de un nuevo enfoque científico}

A lo largo del siglo XX hemos vivido una transformación radical del concepto de conocimiento y del concepto de ciencia. Estamos llegando a la adopción de un nuevo concepto de la racionalidad científica, de un nuevo paradigma epistemológico. El modelo científico positivista — que imperó por más de tres siglos- comenzó a ser cuestionado severamente a principios del siglo XX por los físicos y por los psicólogos de la Gestalt; luego, más tarde - en la segunda década - por los lingüistas, y finalmente - en los años 30, 40, 50 y, sobre todo, en los 60- por los biólogos y los filósofos de la ciencia. Todos, unos tras otros, fueron manifestando su insatisfacción con la racionalidad lineal, unidireccional, y viendo, poco a poco, la necesidad de reemplazar el modelo axiomático de pensar, razonar y demostrar, con su ideal puro lógico-formal o lógico-matemático, con una lógica que diera cabida a la auténtica y más empírica realidad del mundo en que vivimos y con el que interactuamos, de un mundo donde existen inconsistencias reales, incoherencias lógicas y hasta contradicciones conceptuales. 
Esta es la tesis básica que defienden las diferentes orientaciones pospositivistas, las cuales consideran insostenible el modelo reduccionista "variable independiente $\rightarrow$ variable dependiente" ligadas únicamente por una relación causal, y la necesidad de sustituirlo por un modelo sistémico cónsono con la complejidad de las realidades del mundo actual.

El gran físico Erwin Schrödinger, premio Nobel por su descubrimiento de la ecuación fundamental de la mecánica cuántica (base de la física moderna), considera que la ciencia actual nos ha conducido por un callejón sin salida y que la actitud científica ha de ser reconstruida, que la ciencia ha de rehacerse de nuevo (1967). Y Heidegger (1974) sostiene que "por todas partes se han despertado hoy, en las distintas disciplinas, tendencias a poner la investigación sobre nuevos fundamentos" (p. 19).

El modelo de ciencia que se originó después del Renacimiento sirvió de base para el avance científico y tecnológico de los siglos posteriores. Sin embargo, la explosión de los conocimientos, de las disciplinas, de las especialidades y de los enfoques que se ha dado en el siglo XX, y la reflexión epistemológica, encuentran ese modelo tradicional de ciencia no solo insuficiente, sino, sobre todo, inhibidor de lo que podría ser un verdadero progreso tanto particular, como integrado de las diferentes áreas del saber.

Por todo ello conviene enfatizar que esta situación no es algo superficial, ni coyuntural; el problema es mucho más profundo y serio: su raíz llega hasta las estructuras lógicas de nuestra mente, hasta los procesos que sigue nuestra razón en el modo de conceptualizar y dar sentido a las realidades; en consecuencia, este problema desafía nuestro modo de entender, reta nuestra lógica, reclama un alerta, pide mayor sensibilidad intelectual, exige una actitud crítica constante, y todo ello bajo la amenaza de dejar sin rumbo y sin sentido nuestros conocimientos considerados como los más seguros por ser "científicos". El conocimiento no es, en pocas palabras, un reflejo especular de "lo que está allá afuera"; el conocimiento es el resultado de un elaboradísimo proceso de interacción entre un estímulo sensorial (visual, auditivo, olfativo, etc. o un contenido de nuestra memoria) y todo nuestro mundo interno de valores, intereses, creencias, sentimientos, temores, etc.

De esta manera, el problema principal que enfrenta actualmente la investigación y su metodología tiene un fondo esencialmente epistemológico, pues gira en torno al concepto de conocimiento y de ciencia y la respetabilidad científica de sus productos: el conocimiento de la verdad y de las leyes de la naturaleza. De aquí, la aparición, sobre todo en la segunda parte del siglo XX, de las corrientes posmodernistas, las posestructuralistas, el construccionismo, el desconstruccionismo, la Teoría crítica, el análisis del discurso, la desmetaforización del discurso y, en general, los planteamientos que formula la Teoría del conocimiento.

Nuestro objetivo fundamental, aquí, será clarificar e ilustrar que el problema reside en el concepto restrictivo de cientificidad adoptado, especialmente en las ciencias humanas, que mutila la legitimidad y derecho a existir de una gran riqueza de la dotación más típicamente humana, como los procesos que se asientan en el uso de la libertad y de la creatividad. Esta gran riqueza de dotación exige en el investigador, por un lado, una gran sensibilidad en cuanto al uso de métodos, técnicas, estrategias y procedimientos para poder captarla, y, por el otro, un gran rigor, sistematicidad y criticidad, como criterios básicos de la cientificidad requerida por los niveles académicos. 
Debido a los arduos debates epistemológicos durante las cinco primeras décadas del siglo $\mathrm{XX}$, en la década de los años 60 se desarrollan 5 Simposios internacionales sobre filosofía de la ciencia para estudiar a fondo este extremadamente difícil problema que constituía un auténtico cambio de paradigma epistémico. Estos simposios terminan afirmando, especialmente el último (de 1969), que "ha llegado la hora de ir mucho más allá de la imagen estática, instantánea, de las teorías científicas a la que los filósofos de la ciencia se han autolimitado durante tanto tiempo", ya que la concepción heredada, con el positivismo lógico que implica, "ha sido refutada", "es fundamentalmente inadecuada e insostenible y debe sustituirse", ha sufrido "un rechazo general", y por ello "ha sido abandonada por la mayoría de los filósofos de la ciencia” (Suppe, 1979, pp. 16, 89, 145, 149).

Según Echeverría (1989: 25) este último simposio, con estas y otras muchas ideas, "levantó el acta de defunción de la concepción heredada (el positivismo lógico), la cual, a partir de ese momento, quedó abandonada por casi todos los epistemólogos”, debido, como señala Popper, "a sus dificultades intrínsecas insuperables” (1977: 118). En ese ataúd memorable se introdujeron muchas ideas que, sin embargo, siguen circulando en nuestros medios académicos como "conocimientos científicos", cuando en realidad no son más que cadáveres ambulantes. Es muy grande el daño que podemos hacer a nuestros estudiantes por falta de actualización epistemológica y basándonos en una "racionalidad" anacrónica y endiosada (la diosa razón del siglo de las Luces) que, más que una auténtica razón, está constituida por hábitos y hasta rutinas mentales.

Por todo ello, conviene oír la solemne declaración pronunciada más recientemente (1986) por James Lighthill, presidente de la International union of theoretical and applied mechanics, es decir, la Sociedad internacional actual de la mecánica, a cuya orientación ideológica perteneció el mismo Heinrich Hertz, descubridor de las ondas hertzianas y creador fundamental del método científico tradicional.

Aquí debo detenerme y hablar en nombre de la gran fraternidad que formamos los expertos de la mecánica. Somos muy conscientes, hoy, de que el entusiasmo que alimentó a nuestros predecesores ante el éxito maravilloso de la mecánica newtoniana los condujo a hacer generalizaciones en el dominio de la predictibilidad (...), que reconocemos ahora como falsas. Queremos colectivamente presentar nuestras excusas por haber inducido a error a un público culto, divulgando, en relación con el determinismo de los sistemas que satisfacen las leyes newtonianas del movimiento, ideas que, después de 1960, se han demostrado incorrectas (p. 38).

Esta confesión no necesita comentario alguno, pues como dice el lema de la justicia procesal, "a confesión de reo, relevo de pruebas".

\section{El conocimiento y sus procesos mentales}

Según la psicología del pensamiento, nuestra mente puede realizar una percepción y su consiguiente conocimiento de la realidad de tres formas: 1) centrándose en un elemento o parte de la misma (por ejemplo, el ojo, la nariz, etc. de un rostro), 2) captando todo el rostro (el sistema que forman todos sus elementos), y 3) percibiendo la esencia como concepto universal de lo que es la fisonomía del ser humano. 
Es esta última realidad la que busca la ciencia; es este concepto de lo esencial el que fundamenta el progreso y la cultura de las civilizaciones, y a su búsqueda se dedica toda investigación, digna de tal nombre, elaborando síntesis teóricas de las realidades que estudia, ya sea por medio de analogías y metáforas o, también, creando modelos o, incluso, verdaderas teorías.

La primera de las percepciones señaladas arriba, la visión atomística de la realidad, tiene una larga historia. Renè Descartes, en su Discurso del método (1983, orig.1637), expresa una idea rectora que pone como segunda regla: "dividir cada una de las dificultades en tantas partes como sea posible y necesario para mejor resolverlas". Igualmente señala que "la diversidad de nuestras opiniones no viene del hecho que unos seamos más razonables que otros, sino del hecho que conducimos nuestros pensamientos por vías diferentes y no consideramos las mismas cosas". También Newton expresó la gran ley de la gravitación universal con una sola fórmula matemática sintetizando magníficamente con ella las obras de Copérnico y Kepler, e, igualmente, las de Bacon, Galileo y Descartes. Newton, después, supuso que las normas generales que parecen obedecer los cuerpos de tamaño intermedio son también verdad para cada partícula de materia, sea cual sea su clase y tamaño. Las ideas de Descartes y de Newton se mantuvieron, en su esencia, hasta la década de los años 60 del siglo XX, y, más específicamente, hasta 1969, año del V Simposio internacional sobre filosofía de la ciencia ya señalado.

En efecto, Fritjof Capra, físico actual, puntualiza (1992) que la teoría cuántica nos obliga a ver el universo, no como una colección de objetos físicos, sino más bien como una red compleja de relaciones entre las distintas partes de un todo unificado; estos aspectos los veremos más adelante en profundidad.

Popper clarifica esta situación al decir: “en los años veinte comprendí lo que la revolución einsteniana significó para la epistemología: si la teoría de Newton, que estaba rigurosamente probada y que se había corroborado mejor de lo que un científico nunca pudo soñar, se reveló como una hipótesis insegura y superable, entonces no había ninguna esperanza de que cualquier teoría física pudiese alcanzar más que un estatus hipotético, o sea una aproximación a la verdad" (Rivadulla, 1986: 297).

Pero este estado de cosas plantea una interrogante muy seria a nuestra docencia universitaria, que se podría concretar en la siguiente pregunta: ¿hasta dónde los conocimientos que transmitimos están anclados en una sólida y firme base crítica, en un paradigma epistémico coherente y defendible con los mejores argumentos disponibles hoy en día, y hasta qué punto, en cambio, esos "conocimientos" son solo la continuación de la inercia mental que rige gran parte del comportamiento humano? Es posible, entonces, que sigamos llenando nuestras revistas y nuestras hemerotecas con publicaciones de trabajos que no resisten una crítica epistemológica seria y actualizada; es muy posible, también, que sigamos trabajando y divulgando hallazgos que, bien examinados en sus bases de sustentación, no son sino "pseudoinvestigaciones".

Y, más concretamente aún, lo expresa Mary Hesse con las siguientes expresiones: "Doy por suficientemente demostrado que los datos no son separables de la teoría y que su expresión está transida de categorías teoréticas; que el lenguaje de la ciencia teórica es irreductiblemente metafórico e informalizable, y que la lógica de la ciencia es interpretación 
circular, reinterpretación y autocorrección de los datos en términos de teoría, y de la teoría en términos de datos" (en Habermas, 1996: 462).

El método científico tradicional ha seguido la lógica lineal unidireccional, ya sea en una "línea" deductiva como en una inductiva. La línea deductiva la ha seguido principalmente en su utilización en las ciencias formales (lógica y matemática), es decir, en la aplicación (frecuentemente, mala aplicación: no aristotélica) de la lógica clásica, como también en la aplicación de las matemáticas (aritmética, álgebra y geometría); pero la ha seguido igualmente en el campo de las ciencias naturales, especialmente de la física y la química. La lógica lineal deductiva parte de unos primeros principios (lógica filosófica: principio de identidad, de no contradicción, del tercero excluido, "no, si este es bien entendido"), o de un sistema de axiomas, postulados o primitivos, como lo hace en geometría (postulados euclidianos), o en aritmética y álgebra (sistema de axiomas de Peano; ver Frey, 1972: 67), o incluso, de un solo principio fundamental, como lo hace Heinrich Hertz partiendo del principio de inercia en su magistral y paradigmática obra Principios de la mecánica (1956, orig. 1894), con que puso las bases teóricas del método científico tradicional. Esta lógica dirige la mente humana para hacerle ver (demostrando) que un determinado teorema o proposición ya está implícito en los axiomas, postulados o principios fundamentales, aceptados como base, los cuales son evidentes de por sí y, por lo tanto, no necesitan demostración.

La lógica lineal inductiva, al contrario, sigue el camino inverso: de muchas constataciones particulares, generaliza hacia una conclusión universal. Pero la constatación de muchos casos en una muestra (por muy numerosos y relevantes que sean) nunca nos da la certeza de su posible aplicación a todos los casos que constituyen el universo del cual se extrajo la muestra. De aquí la debilidad de la lógica inductiva. Por ello, siempre concluye con unos resultados sujetos a un nivel de probabilidad de error aceptable: $1 \%, 5 \%$, etc.

La mayor debilidad de la lógica lineal es su irrealidad, es decir, su lejanía de la realidad concreta, especialmente si se trata de problemas de la ciencias humanas, donde no se da únicamente una variable independiente, una dependiente y una relación de causalidad, sino que siempre entran en juego docenas de variables que no son lineales, ni unidireccionales, ni solamente causales, sino variables que interactúan mutuamente y entre las cuales se dan toda clase y tipo de relaciones: de causa, condición, contexto, soporte, aval, secuencia, asociación, propiedad, contradicción, función, justificación, medio, etc.

Popper (1985) dice que "en contra del empirismo inglés de Locke, Berkeley y Hume que estableció la tradición consistente en tomar la percepción sensible como paradigma fundamental, si no único, de experiencia consciente y de experiencia cognoscitiva, hay que reconocer que propiamente no hay datos sensoriales (...), que lo que la mayoría de las personas considera un simple dato es de hecho el resultado de un elaboradísimo proceso (...) de toma y dame entre los estímulos externos y la actividad del cerebro" (pp. 140, 483).

\section{Complejidad y transdisciplinariedad}

Los términos complejidad y transdisciplinariedad se usan hoy día frecuentemente en los medios académicos en su relación con la epistemología, y algo similar sucede con el 
término "paradigma"; sin embargo, no existe una definición compartida de los mismos, razón por cual la comunicación entre los diferentes usuarios de los mismos falla, a veces, en forma más que grave.

Tenemos que tener muy presente que no basta una definición etimológica de los términos en uso, ni tampoco una semántica, pues, frecuentemente, es su uso, su pragmática - es decir, la que usan los hablantes de una cultura o disciplina- la que ordinariamente juega el papel principal en la determinación del significado de un término, pues en la práctica el significado es algo convencional ya que, como nos recuerdan lingüistas como Ferdinand de Saussure (1954), se debe señalar que no existe conexión alguna entre el signo y su referente, es decir, que las palabras tienen un origen arbitrario o convencional. Recordemos también lo que nos dice Heisenberg con relación a las partículas en física cuántica: que son todo menos partículas, es decir, partecitas del átomo, ya que frecuentemente son simples relaciones y, a veces, relaciones de relaciones; y esta aclaratoria la aplica al $50 \%$ de los términos de la física newtoniana.

Lo que generalmente llamamos "realidades complejas, hipercomplejas o transcomplejas" y procesos de estudio "transdisciplinarios" están referidos, básicamente, a los procesos mentales fundamentales, gestálticos y estereognósicos, con los cuales conceptualizamos y expresamos las totalidades en forma integral y sistémica. Todos estos términos técnicos, que a veces confunden nuestro pensamiento, pudiéramos decir que no son, ni expresan algo esencialmente diferente de lo que hace nuestra mente cuando, inconscientemente, aprecia la "realidad integral" en forma holista y sistémica.

Como el nivel superior de nuestras actividades mentales está constituido por características, propiedades y atributos definidores especiales, nunca se podrá reducir a niveles inferiores y explicar en sus términos atomísticos (vicio del reduccionismo): las fuerzas físicas, por ejemplo, no serán suficientes para explicar la fuerza que mueve la economía o los impulsos sexuales o lo que lleva a la gente a suicidarse; los componentes químicos de la pintura nunca explicarán la expresión de la Monna Lisa, ni los componentes físicos de la obra, el significado de Hamlet. Como decía Whitehead, si se quieren conocer los principios básicos de la existencia hay que utilizar lo superior para iluminar lo inferior y no al revés, como hace la reflexión reduccionista corriente.

La ciencia tradicional ha prestado, sin duda alguna, muchos servicios al hombre: le ha ayudado a superar mucha pobreza, enfermedades, trabajo deshumanizante y, en general, a alargar su vida. Pero el querer llevar el método científico a todos los campos, ha hecho que, hablando de refracción de ondas luminosas, pigmentación y colores espectrales, la ciencia haya anulado las puestas de sol, los paisajes y los arcoiris; que, tratando de ser científicos, los estructuralistas hayan desfigurado la prosa y la poesía; que, analizando computacionalmente el Nuevo Testamento, los estudiosos bíblicos destruyan la fe y la conciencia religiosa.

En efecto, si fijamos nuestra atención en el montón de ladrillos y piedras nunca captaremos la belleza de una catedral gótica; si detenemos nuestra atención en la blusa, zapatos o maquillaje de una dama nunca captaremos la elegancia de su presencia. Sabemos, nada menos que desde Aristóteles, que "el todo es más que la suma de sus partes", pues "el todo" es la interrelación sistémica de todas las partes y la anomalía de una sola rompe 
la elegancia del conjunto. Es más, esto que parece tan sencillo y cotidiano, implica unos procesos mentales gestálticos y estereognósicos que exigen un paradigma sistémico para su cabal comprensión, como veremos más adelante.

Entremos más a fondo en el verdadero problema. Los fenómenos de la vida y la posibilidad del hombre de interactuar con ellos han creado una fuerte y amplia discusión metodológica. No es nada fácil comprender, aceptar y llevar la lógica de una determinada disciplina a las mentes de los que cultivan otra muy diferente. Sin embargo, no se trata de eso: se trata de un encuentro y diálogo académicos que se interfecundan.

En general, existe un punto muy controversial: se considera que los instrumentos de investigación propios de las ciencias naturales (física, química y, también, matemática) no son lo suficientemente exhaustivos en la búsqueda de la complejidad biológica, psicológica, sociológica y otras ciencias humanas, ya que estas ciencias son muy "particulares". Es natural que un enfoque metodológico básicamente diferente conduzca a la formulación de paradigmas científicos contrastantes. Interpretar las estructuras de estas ciencias como líneas matemáticas significa negar el concepto mismo con que se definen, significa negar el valor del comportamiento como factor evolutivo y el de la influencia del ambiente sobre el sujeto mutante. No se puede considerar un sujeto viviente cualquiera como una cifra de un sistema algorítmico, ya que son sistemas abiertos profundamente interrelacionados con el ambiente en que viven. Tenemos aquí, por consiguiente, el uso de lógicas epistémicas particulares.

¿En qué consiste esta dimensión cualitativa y sistémica de la ciencia? La previsión probabilística, debido precisamente al alto número de factores que determinan el fenómeno de los seres vivos, no agota su estudio. La física y la matemática no pueden ser utilizadas y concebidas como parámetros adecuados de las ciencias de la vida; los mismos físicos tuvieron que abandonar, a principios del siglo XX, el paradigma mecanicista al llegar al nivel submicroscópico. Esto no significa negar el valor de estas disciplinas, sino subrayar su dimensión no exhaustiva en la investigación de la vida (Schrödinger, 1967), ya que su estructura se define con conceptos propios, extremadamente peculiares, como la teleonomía, la invarianza, la especie, el ecosistema, el organismo, etcétera, dentro de los cuales están insertados otros conceptos que conforman un sistema abierto en continua evolución y cambio, como los conceptos de autoorganización, automantenimiento, autotransformación, autorenovación y autotransferencia, todos los cuales configuran una especie de autopoiesis, es decir, una especie de autocreación. Todos estos conceptos, centrados en la vida pueden estar muy alejados de la mente, por ejemplo, de un físico, de un químico e, incluso, de un abogado.

Sin embargo, sobre estos conceptos construyen las ciencias de la vida, y las ciencias humanas, en general, sus propias coordenadas gnoseológicas; es decir que caminan por sendas heurísticas propias. De aquí, la necesidad de identificar una lógica no numérica, como guía del proceso heurístico, es decir, la dimensión o estructura sistémico-cualitativa de la ciencia.

En síntesis, los diferentes niveles en que se nos presenta la realidad, en todos los campos, pero de una manera especial la realidad de los seres vivos, exige también diferentes niveles de la lógica a aplicar y, en nuestro caso, una dialógica transdisciplinaria y unos métodos 
también transdisciplinarios; todo lo cual nos introduce en el paradigma sistémico, pues como dice von Bertalanffy: "desde el átomo hasta la galaxia vivimos en un mundo de sistemas" (1981: 47).

\section{La "experiencia de verdad" transdisciplinaria}

En el ámbito de la experiencia total humana existe, además, una "experiencia de verdad" (Gadamer, 1984: 24-25), una vivencia con certeza inmediata, como la experiencia de la filosofía, del arte y de la misma historia, que son formas de experiencia en las que se expresa una verdad que no puede ser verificada con los medios de que dispone la metodología científica tradicional. En efecto, esta metodología usa, sobre todo, lo que Eccles (1985) llama el etiquetado verbal propio del hemisferio izquierdo, mientras que la experiencia total requiere el uso de procesos estereognósicos propios del hemisferio derecho: este hemisferio - dice Eccles- "funciona como un cerebro muy superior, con una refinada habilidad de estereognosia, reconocimiento de patrones y copia” (pp. 354, 520, 521).

Gadamer (1984) señala que en los textos de los grandes pensadores, como Platón, Aristóteles, Marco Aurelio, San Agustín, Leibniz, Kant o Hegel, "se conoce una verdad que no se alcanzaría por otros caminos; aunque esto contradiga al patrón de investigación y progreso con que la ciencia acostumbra a medirse". Igual vivencia se experimentaría en la "experiencia del arte", vivencia que no se puede pasar por alto, ya que "en la obra de arte se experimenta una verdad que no se logra por otros medios, y es lo que hace el significado filosófico del arte que se afirma frente a todo razonamiento". Pero es nuestro deber, añade este autor, "intentar desarrollar un concepto de conocimiento y de verdad que responda al conjunto de nuestra experiencia hermenéutica”. Como vemos, Gadamer está apuntando aquí hacia una nueva forma heurística que camina en la dirección de la transdisciplinariedad (pp. 24, 139).

Continúa aclarando Gadamer cómo esta experiencia vivencial — que "como vivencia queda integrada en el todo de la vida y, por lo tanto, el todo se hace también presente en ella" - es un auténtico conocimiento; es decir, mediación de verdad, no ciertamente como conocimiento sensorial, conceptual o racional, de acuerdo a la ciencia y según el concepto de realidad que sustentan las ciencias de la naturaleza, sino como una pretensión de verdad diferente de la ciencia, aunque seguramente no subordinada, ni inferior a ella. Por esto cree que "la oposición entre lo lógico y lo estético se vuelve dudosa" (ibid. pp. 107, 139, 656).

\section{ASPECTOS FUNDAMENTALES DE UNA EPISTEMOLOGÍA ACTUALIZADA}

\section{El paradigma sistémico}

Como dice Beynam (1978): "actualmente vivimos un cambio de paradigma en la ciencia, tal vez el cambio más grande que se ha efectuado hasta la fecha (...) y que tiene la ventaja adicional de derivarse de la vanguardia de la física contemporánea”. Está emergiendo un nuevo paradigma que afecta a todas las áreas del conocimiento. La nueva ciencia no 
rechaza las aportaciones de Galileo, Descartes o Newton, sino que las integra en un contexto mucho más amplio y con mayor sentido, en un paradigma sistémico.

Ahora bien, bajo el punto de vista ontológico, ¿cómo se nos presenta la realidad, en general, de nuestro universo?, ¿pueden reducirse los seres que nos rodean a su dimensión lineal, cuantitativa? Nuestro universo está constituido básicamente por sistemas no-lineales en todos sus niveles: físico, químico, biológico, psicológico y sociocultural.

"Si observamos nuestro entorno vemos que estamos inmersos en un mundo de sistemas. Al considerar un árbol, un libro, un área urbana, cualquier aparato, una comunidad social, nuestro lenguaje, un animal, el firmamento, en todos ellos encontramos un rasgo común: se trata de entidades complejas, formadas por partes en interacción mutua, cuya identidad resulta de una adecuada armonía entre sus constituyentes, y dotadas de una sustantividad propia que transciende a la de esas partes; se trata, en suma, de lo que, de una manera genérica, denominamos sistemas" (Aracil, 1986: 13).

Según el físico Fritjof Capra (1992), la teoría cuántica demuestra que todas las partículas se componen dinámicamente unas de otras de manera autoconsistente y, en ese sentido, puede decirse que "contienen" la una a la otra, que se "definen" la una con la otra. De esta forma, la física (la nueva física) es un modelo de ciencia para los nuevos conceptos y métodos de otras disciplinas. En el campo de la biología, Dobzhansky ha señalado que el genoma, que comprende tanto genes reguladores como operantes, trabaja como una orquesta y no como un conjunto de solistas. También Köhler (para la psicología) solía decir que "en toda estructura dinámica (o sistema) cada parte conoce dinámicamente a cada una de las otras". Y Ferdinand de Saussure (para la lingüística: 1954) afirmaba que "el significado y valor de cada palabra está en las demás", que el sistema es "una totalidad organizada, hecha de elementos solidarios que no pueden ser definidos más que los unos con relación a los otros en función de su lugar en esta totalidad".

Si la significación y el valor de cada elemento de una estructura dinámica o sistema está íntimamente relacionado con los demás, si todo es función de todo, y si cada elemento es necesario para definir a los otros, no podrá ser visto, ni entendido "en sí" en forma aislada, sino a través de la posición y de la función o papel que desempeña en esa estructura dinámica o sistema.

A este respecto, y refiriéndose a la sociología, dice muy bien Th.W. Adorno: "parece innegable que el ideal epistemológico de la elegante explicación matemática, unánime y máximamente sencilla, fracasa allí donde el objeto mismo, la sociedad, no es unánime, ni es sencillo, ni viene entregado de manera neutral al deseo o a la conveniencia de la formalización categorial, sino que es, por el contrario, bien diferente a lo que el sistema categorial de la lógica discursiva espera anticipadamente de sus objetos. La sociedad es contradictoria y, sin embargo, determinable; racional e irracional a un tiempo; es sistema y es ruptura; naturaleza ciega y mediación por la conciencia. A ello debe inclinarse el proceder todo de la sociología. De lo contrario, incurre, llevada de un celo purista contra la contradicción, en la más funesta de todas: en la contradicción entre su estructura y la de su objeto" (Mardones, 1991: 331). 
Cabe, entonces, la pregunta: ¿cuál es la "razón” en que se apoya la tendencia a matematizar toda realidad, a expresarla en un lenguaje matemático inadecuado, insuficiente y reductivo, imprimiendo sus formas e idealizando y empobreciendo las disciplinas renuentes a ello? Eichner (1989) nos da una respuesta: "la objeción aquí no es al uso de las matemáticas o al matematicismo de la economía. Es (....) al modo cómo las matemáticas han sido usadas para dar una fachada pseudocientífica a un cuerpo de la teoría, el cual no puede satisfacer ninguna de las pruebas empíricas mediante las cuales la ciencia se diferencia de la mera superstición o de la ideología pura” (p. 34).

La naturaleza íntima de los sistemas o estructuras dinámicas, en efecto, su entidad esencial, está constituida por la relación entre las partes, y no por estas, tomadas en sí. La relación es una entidad emergente, nueva. El punto crucial y limitante de nuestra matemática tradicional, por ejemplo, se debe a su carácter abstracto, a su incapacidad de captar la entidad relacional. La abstracción es la posibilidad de considerar un objeto o un grupo de objetos desde un solo punto de vista, prescindiendo de todas las restantes particularidades que pueda tener

El enfoque sistémico es indispensable cuando tratamos con estructuras dinámicas o sistemas que no se componen de elementos homogéneos y, por lo tanto, no se le pueden aplicar las cuatro leyes que constituyen nuestra matemática actual sin desnaturalizarlos, la ley aditiva de elementos, la conmutativa, la asociativa y la distributiva de los mismos, pues, en realidad, no son "elementos homogéneos", ni agregados, ni "partes", sino constituyentes de una entidad superior; las realidades sistémicas se componen de elementos o constituyentes heterogéneos y son lo que son por su posición o por la función que desempeñan en la estructura o sistema total; es más, el buen o mal funcionamiento de un elemento repercute o compromete el funcionamiento de todo el sistema: ejemplos de ello los tenemos en todos los seres vivos y aun en la tecnología, como el estrepitoso fracaso del Challenger o del Ariane V, debidos, respectivamente, a una superficie exterior no cuidada o a los "tiempos" de una computadora. En general, podríamos señalar, como una especie de referente clave, que la matemática trabaja bien con objetos constituidos por elementos homogéneos y pierde su capacidad de aplicación en la medida en que estos son de naturaleza heterogénea, donde entra en acción lo cualitativo y sus relaciones.

El gran biólogo Ludwig von Bertalanffy dice - como ya señalamos- que desde el átomo hasta la galaxia vivimos en un mundo de sistemas y señaló (en 1972) que para entender matemáticamente, por ejemplo, los conceptos biológicos de diferenciación, desarrollo, equifinalidad, totalidad, generación, etc. (todos sistémicos) necesitaríamos unas "matemáticas gestálticas", en las que fuera fundamental, no la noción de cantidad, sino la de relación, forma y orden.

Hoy en día, ya se han desarrollado mucho estas matemáticas. Se conocen con los nombres de "matemáticas de la complejidad", "teoría de los sistemas dinámicos" o "dinámica no-lineal", y trabajan con centenares de variables interactuantes e intervinientes durante los procesos con la cuarta dimensión "tiempo". Se trata de unas "matemáticas más cualitativas que cuantitativas". En ellas se pasa de los objetos a las relaciones, de las cantidades a las cualidades, de las substancias a los patrones. Su práctica es posible gracias a los ordenadores de alta velocidad que pueden ahora resolver problemas complejos, no- 
lineales (con más de una solución), antes imposibles, graficar sus resultados en curvas y diagramas para descubrir patrones cualitativos (sin ecuaciones ni fórmulas), guiados por los llamados "patrones atractores", es decir, que exhiben tendencias (ver Capra, 2003, especialmente cap. 6).

El pensamiento sistémico comporta, además, un cambio de la ciencia objetiva a la ciencia epistémica; es decir, se tiene en cuenta la posición personal del sujeto investigador, como el físico tiene en cuenta la temperatura previa del termómetro que usa.

La comprensión de toda entidad que sea un sistema o una estructura dinámica requiere el uso de un pensamiento o una lógica dialécticos, no le basta la relación cuantitativoaditiva y ni siquiera es suficiente la lógica deductiva ya que aparece una nueva realidad emergente que no existía antes, y las propiedades emergentes no se pueden deducir de las premisas anteriores. Estas cualidades no están en los elementos sino que aparecen por las relaciones que se dan entre los elementos: así surgen las propiedades del agua, que no se dan ni en el oxígeno ni en el hidrógeno por separado; así aparece o emerge el significado al relacionarse varias palabras en una estructura lingüística; así emerge la vida por la interacción de varias entidades físico-químicas, etc.

El principio de exclusión del físico cuántico Wolfgang Pauli, por su parte, estableció desde 1925 que las "leyes-sistemas" no son derivables de las leyes que rigen a sus componentes. Las propiedades que exhibe, por ejemplo, un átomo en cuanto un todo, se gobiernan por leyes no relacionadas con aquellas que rigen a sus "partes separadas"; el todo es entendido y explicado por conceptos característicos de niveles superiores de organización. Y este principio se extiende a todos los sistemas o estructuras dinámicas que constituyen nuestro mundo: sistemas atómicos, sistemas moleculares, sistemas celulares, sistemas biológicos, psicológicos, sociológicos, culturales, etc. La naturaleza de la gran mayoría de los entes o realidades es un todo polisistémico que se rebela cuando es reducido a sus elementos. Y se rebela, precisamente, porque así, reducido, pierde las cualidades emergentes del "todo" y la acción de estas sobre cada una de las partes.

Por todo ello, nunca entenderemos, por ejemplo, la pobreza de una familia, de un barrio, de una región o de un país en forma aislada, desvinculada de todos los demás elementos con que está ligada, como tampoco entenderemos el desempleo, la violencia o la corrupción, por las mismas razones; y menos sentido aun tendrá la ilusión de querer solucionar alguno de estos problemas con simples medidas aisladas.

En consecuencia, cada disciplina deberá hacer una revisión, una reformulación o una redefinición de sus propias estructuras lógicas individuales, que fueron establecidas aislada e independientemente del sistema total con que interactúan, ya que sus conclusiones, en la medida en que hayan cortado los lazos de interconexión con el sistema global de que forman parte, serán parcial o totalmente inconsistentes. Esto equivale a decir que debemos pasar de los planes de estudio monodisciplinares a planes de estudio multidisciplinarios, interdisciplinarios y transdisciplinarios. 


\section{Conocimiento tácito: aprehensión en sí y aprehensión subsidiaria}

Si nos adentramos más en el fenómeno "partes-todo" y enfocamos más de cerca su aspecto gnoseológico, diremos, con la psicología de la Gestalt, que hay dos modos de aprehensión intelectual de un elemento que forma parte de una totalidad. Michael Polanyi (1966) lo expresa de la siguiente manera: “... no podemos comprender el todo sin ver sus partes, pero podemos ver las partes sin comprender el todo (...). Cuando comprendemos como parte de un todo a una determinada serie de elementos, el foco de nuestra atención pasa de los detalles hasta ahora no comprendidos a la comprensión de su significado conjunto. Este pasaje de la atención no nos hace perder de vista los detalles, puesto que solo se puede ver un todo viendo sus partes, pero cambia por completo la manera cómo aprehendemos los detalles. Ahora los aprehendemos en función del todo en que hemos fijado nuestra atención. Llamaré a esto aprehensión subsidiaria de los detalles, por oposición a la aprehensión focal que emplearíamos para atender a los detalles en sí, no como partes del todo" (pp. 22-23).

En este campo, Polanyi sigue de cerca las ideas de Merleau-Ponty sobre el concepto de estructura. En efecto, Merleau-Ponty (1976) afirma que las estructuras no pueden ser definidas en términos de realidad exterior, sino en términos de conocimiento, ya que son objetos de la percepción y no realidades físicas; por eso, las estructuras no pueden ser definidas como cosas del mundo físico, sino como conjuntos percibidos $\mathrm{y}$, esencialmente, consisten en una red de relaciones percibidas que, más que conocida, es vivida (pp. 204, 243).

Como científico y filósofo, Polanyi trata de esclarecer en múltiples estudios (1966, 1968, 1969) lo que estas ideas implican, y llega así a su teoría del conocimiento tácito y a la lógica de la inferencia tácita. Estos son poderes extraordinarios (usados ordinariamente) que posee el ser humano, acerca de los cuales apenas tiene conciencia precisamente porque su dinámica es inconsciente o actúa a un nivel subliminal. En efecto, la mayor parte del proceso mental es inconsciente, como constatamos al hablar sin pensar en cada palabra, ni en su articulación vocal. Por ello, Kant dice que "ese proceso está tan escondido en el alma humana que muy difícilmente podemos imaginar el secreto que emplea aquí la naturaleza" (1787, A.141; ver Polanyi, 1969: 105).

Así, cuando fijamos aisladamente el foco de nuestra atención en los detalles de una realidad cualquiera nos resultan incomprensibles y sin sentido; en cambio, cuando nuestra atención va más allá de ellos y se dirige a la entidad emergente de la que ellos forman parte y a la cual contribuyen resultan llenos de significado, sentido y explicación. Un ejemplo sencillo lo tenemos en el estereoscopio, en el que la emergencia de una tercera dimensión solo aparece cuando nos olvidamos de las dos imágenes individuales y proyectamos nuestra visión más allá de ellas.

Ahora bien, el estudio de entidades emergentes como estas requiere el uso de una lógica no deductiva; requiere una lógica dialéctica en la cual las partes son comprendidas desde el punto de vista del todo. Dilthey (1900) llama círculo hermenéutico a este proceso interpretativo, al movimiento que va del todo a las partes y de las partes al todo tratando de buscarle el sentido. En este proceso, el significado de las partes o componentes está determinado por el conocimiento previo del todo, mientras que nuestro conocimiento del 
todo es corregido continuamente y profundizado por el crecimiento de nuestro conocimiento de los componentes.

En esta línea de pensamiento, es importante destacar la obra de Gadamer (1976), en la cual elabora un modo de pensar que va más allá del objetivismo y relativismo y que explora "una noción enteramente diferente del conocimiento y de la verdad". En efecto, la lógica dialéctica supera la causación lineal y unidireccional explicando los sistemas auto-correctivos, de retro-alimentación y pro-alimentación, los circuitos recurrentes y aun ciertas argumentaciones que parecieran ser "circulares". Por otra parte, la lógica dialéctica goza de un sólido respaldo filosófico, pues se apoya en toda la filosofía dialéctica de Hegel, que es, sin duda, uno de los máximos exponentes de la reflexión filosófica a lo largo de toda la historia de la humanidad.

Hegel (1966) había precisado muy bien "este movimiento dialéctico", como lo llama él: donde el "ser en sí" pasa a ser "un ser para la conciencia" y "lo verdadero es el 'ser para ella' de ese 'ser en sí'”. Pero entre la pura aprehensión de ese objeto en sí y la reflexión de la conciencia sobre sí misma "yo me veo repelido hacia el punto de partida y arrastrado de nuevo al mismo ciclo, que se supera en cada uno de sus momentos y como totalidad, pues la conciencia vuelve a recorrer necesariamente ese ciclo, pero, al mismo tiempo, no lo recorre ya del mismo modo que la primera vez" (pp. 58-59, 74-75), es decir, que se va elevando, en forma de una espiral, hacia una comprensión cada vez más completa.

\section{Aporte de la neurociencia actual}

Entre los aportes de la neurociencia actual es de máxima importancia el que esclarece el proceso de atribución de significados. Así, por ejemplo, los estudios sobre la transmisión neurocerebral nos señalan que, ante una sensación visual, auditiva, olfativa, etc., antes de que podamos decir "es tal cosa", se da un ir y venir, entre la imagen o estímulo físico respectivos y el centro cerebral correspondiente, de cien y hasta mil veces, dependiendo del tiempo empleado. Cada uno de estos "viajes" de ida y vuelta tiene por finalidad ubicar o insertar los elementos de la imagen o estímulo sensible en diferentes contextos de nuestro acervo mnemónico, buscándole un sentido o un significado. Pero este sentido o significado será muy diferente de acuerdo a ese "mundo interno personal" y la respectiva estructura en que se ubica: valores, actitudes, creencias, necesidades, intereses, ideales, temores, etc., Popper y Eccles (1985: 483-484).

Pero el mundo en que hoy vivimos se caracteriza por sus interconexiones a un nivel global en el que los fenómenos físicos, biológicos, psicológicos, sociales y ambientales son todos recíprocamente interdependientes. Para describir este mundo de manera adecuada necesitamos una perspectiva más amplia, holista, sistémica y ecológica que no nos pueden ofrecer las concepciones reduccionistas del mundo ni las diferentes disciplinas aisladamente; necesitamos una nueva visión de la realidad, un nuevo "paradigma", es decir, una transformación fundamental de nuestro modo de pensar, de nuestro modo de percibir y de nuestro modo de valorar.

Un conocimiento de algo, sin referencia y ubicación en un estatuto epistemológico que le dé sentido y proyección, queda huérfano y resulta ininteligible; es decir, que ni siquiera 
sería conocimiento. En efecto, conocer es siempre aprehender un dato en una cierta función y bajo una cierta relación, en tanto significa algo dentro de una determinada estructura.

Si el conocimiento se entiende como articulación de toda una estructura epistémica, nadie ni nada podrá ser eximido - llámese investigación, programa, profesor o alumno-, de afrontar los arduos problemas que presenta la epistemología crítica. Lo contrario sería convertir a nuestros alumnos en simples autómatas que hablan de memoria y repiten ideas y teorías o aplican métodos y técnicas entontecedores y hasta cretinizantes, con los cuales ciertamente colapsarán y por los cuales podrían ser arrastrados hacia el vacío cuando una vuelta de la historia, como la que hemos presenciando no hace mucho en los países de la Europa Oriental, mueva los fundamentos epistémicos de todo el edificio. A esto se debe el alerta de la UNESCO, desde hace varios años, sobre este grave problema y su solicitud de que se revisen los planes de estudio de todas las carreras.

\section{El conocimiento como realidad emergente}

El ser humano es superior a los animales, no por la riqueza de su información sensorial, ya que la mayoría de los animales poseen una agudeza visual, auditiva, olfativa, etc. muy superior a la del hombre, sino por su capacidad de relacionar, interpretar y teorizar con esa información. Franz Brentano dice que la imagen mental se forma a través de un conocimiento tácito de las representaciones sensoriales dotándolas de un significado individual propio.

La inteligencia humana tiene una propensión natural innata a buscar regularidades y la capacidad básica de ordenar las cosas, según sean semejantes o diferentes, de acuerdo con su naturaleza y características. Esta actividad mental está en acción continuamente y puede sorprendernos con sus hallazgos tanto por la mañana como por la tarde, en la noche como en el mismo sueño.

El método básico de toda ciencia es la observación de los datos y de los hechos, y la interpretación de su significado. La observación y la interpretación son inseparables: resulta inconcebible que una se obtenga en total aislamiento de la otra. Toda ciencia trata de desarrollar técnicas especiales para efectuar observaciones sistemáticas y garantizar la interpretación.

Pero nuestra observación no está forzada simplemente por lo que tengamos ante los ojos o, incluso, en la retina. A los griegos, por ejemplo, les impresionó la coraza o caparazón que tenían algunos animales y los llamaron quelonios (acorazados); los mismos animales impresionaron a los romanos por sus patas torcidas y, así, los llamaron tortugas (patituertos); ninguna categoría reflejará todo lo real: ¿quelonios griegos o tortugas romanas? ¿De qué depende que nos impresionemos por cosas diferentes? La respuesta a esta pregunta hinca sus raíces nada menos que en la matriz epistémica de que hablamos en otras publicaciones nuestras $(1997,1999,2004,2008)$. También puede depender de la ideología profesada: ¿combatientes o terroristas?, ¿retenidos o secuestrados? 


\section{Repensando la ciencia}

Newton, en su humildad, dijo que "si él había logrado ver más lejos que otros, era porque se había subido sobre los hombros de gigantes", aludiendo con ello a las investigaciones de Copérnico, Galileo, Képler y otros científicos.

En las últimas cinco décadas se han ido dando las condiciones necesarias y suficientes para que todo investigador serio y de reflexión profunda pueda, a través de las bibliotecas, las revistas, los congresos y el uso de la "biblioteca universal" que es el uso de Internet, subirse sobre los hombros de docenas de pensadores eminentes. Y, desde esa atalaya, le es posible divisar grandes coincidencias de ideas y marcadas líneas confluyentes de un nuevo modo de pensar, de una nueva manera de mirar las cosas, de una nueva racionalidad científica y, en síntesis, de una nueva ciencia. Esta ciencia presenta notables diferencias con el modo de pensar tradicional, clásico, lógico-positivista.

La complejidad de las nuevas realidades emergentes durante el siglo XX, su fuerte interdependencia y sus interacciones ocultas, por una parte y, por la otra, el descubrimiento de la riqueza y dotación insospechada de la capacidad creadora y de los procesos cognitivos del cerebro humano, postulan una nueva conciencia y un paradigma de la racionalidad acorde con ambos grupos de realidades.

Nuestro aparato conceptual clásico — que creíamos riguroso, por su "objetividad", determinismo, lógica formal y verificación- resulta corto, insuficiente e inadecuado para simbolizar o modelar realidades que se nos han ido imponiendo, ya sea en el mundo subatómico de la física y en el funcionamiento de nuestro cerebro, como en el de las ciencias de la vida y en las ciencias sociales. Para representarlas adecuadamente necesitamos conceptos muy distintos de los tradicionales y mucho más interrelacionados, capaces de darnos explicaciones globales, unificadas y sistémicas.

En fin de cuentas, eso es lo que somos también cada uno de nosotros mismos: un "todo físicoquímico-biológico-psicológico-social-cultural-espiritual" que funciona maravillosamente y que constituye nuestra vida y nuestro ser. Por esto, el ser humano es la estructura dinámica o sistema integrado más complejo de todo cuanto existe en el universo.

Estamos poco habituados todavía al pensamiento "sistémico-ecológico". El pensar con esta categoría básica cambia en gran medida nuestra apreciación y conceptualización de la realidad. Nuestra mente no sigue solo una vía causal, lineal y unidireccional, sino también y, a veces sobre todo, un enfoque modular, estructural, dialéctico, gestáltico, estereognósico, inter y transdisciplinario, donde todo afecta e interactúa con todo, donde cada elemento no solo se define por lo que es o representa en sí mismo, sino, y especialmente, por su red de relaciones con todos los demás.

En las últimas décadas, la nueva física y la reciente neurociencia nos ofrecen unos "hechos desafiantes" como los siguientes:

- la información entre partículas subatómicas circula de maneras no conformes con las ideas clásicas del principio de causalidad;

- una partícula, al cambiar, modifica instantáneamente a otras a distancia sin señales ordinarias que se propaguen dentro del espacio-tiempo; 
- esa transferencia de información va a una velocidad superior a la de la luz;

- esta información sigue unas coordenadas temporales (hacia atrás y hacia adelante en el tiempo);

- el observador no solo afecta al fenómeno que estudia, sino que en parte también lo crea con su pensamiento al emitir este unas partículas (los psitrones) que interactúan con el objeto;

- nada en el universo está aislado y todo lo que en él convive está, de un modo u otro, interconectado mediante un permanente, instantáneo y hasta sincrónico intercambio de información.

Estos y otros muchos hechos no son imaginaciones de "visionarios", ni solo hipotéticas elucubraciones teóricas, sino conclusiones de científicos de primer plano que demuestran sus teorías con experimentos y pruebas en los aceleradores de partículas y en las cámaras de niebla, y lo hacen con centenares de páginas de sólidos argumentos y hasta de complejos cálculos matemáticos (Racionero y Medina, 1990).

La ciencia occidental avanza cada vez más hacia un cambio de paradigma de proporciones sin precedentes, que cambiará nuestro concepto de la realidad y de la naturaleza humana. En este nuevo paradigma deben tener cabida, ubicación y sistematización todos los conocimientos bien establecidos, ya sea que provengan de la física cuántica y relativista, de la neurociencia, de la parapsicología, del estudio de las estructuras disipativas, de la holografía o de cualquier otra fuente cognoscitiva, y todos formarían un macrosistema integrado: que reflejaría aquella armonía del universo, la cual —según revela Einstein — le guió a él hacia el descubrimiento de la Teoría general de la relatividad. En efecto, podríamos incluso decir que los grandes hallazgos científicos del siglo XX son una aplicación de esta teoría a las diferentes manifestaciones de la naturaleza, a las distintas disciplinas y sus variadas áreas del saber.

\section{Sinergia entre la ciencia, el arte y la ética}

Para muchos científicos, como por ejemplo el mismo Einstein, la ciencia no busca tanto el orden y la igualdad entre las cosas cuanto unos aspectos todavía más generales del mundo en su conjunto, tales como "la armonía", "la simetría", "la belleza", y "la elegancia”, aun a expensas, aparentemente, de su adecuación empírica. En efecto, Hans Reichenbach (miembro del círculo de Viena) reporta una conversación que tuvo con Einstein: "cuando yo -dice él一, en cierta ocasión, le pregunté al profesor Einstein cómo encontró la Teoría de la relatividad, él me respondió que la encontró porque estaba muy fuertemente convencido de la armonía del universo" (en Rogers, 1980: 238). Es más, parece que alguna vez, después de ciertas intuiciones sobre la estructura del universo, se le oyó decir en privado: “ah, viejo (refiriéndose a Dios), ya descubrí tu secreto del universo" (Clark, 1972).

Este concepto de la "armonía”, típicamente estético, liga mucho la ciencia, como él la entendía, con el arte. Cuando Einstein, refiriéndose a la teoría cuántica, dice que "tal teoría no le gusta", que "no le gustan sus elementos", que "no le gustan sus 
implicaciones", etc., su asistente personal de investigación lo interpreta aclarando que "su enfoque (el de Einstein) tiene algo en común con el de un artista; que ese enfoque busca la simplicidad y la belleza (...); que su método, aunque está basado en un profundo conocimiento de la física, es esencialmente estético e intuitivo (...); que, excepto por el hecho de ser el más grande de los físicos desde Newton, uno podría casi decir que Einstein no era tanto un científico cuanto un artista de la ciencia" (Clark, 1972: 648-650).

Por esta misma razón se dice que la belleza es nombrada hoy día más por los físicos que por los críticos de arte. En efecto, mucho antes de que los físicos y, en general, los científicos tomaran conciencia de la importancia del arte como instrumento cognoscitivo, el arte y la literatura ofrecieron soluciones, especialmente a los problemas humanos, en mayor sintonía con su propia naturaleza; es decir, intuiciones más orgánicas, más completas y, por consiguiente, también más verdaderas.

El gran físico cuántico danés Niels Bohr (amigo y, en ciertos temas, opositor de Einstein) afirmaba que "cuando se trata de átomos, el lenguaje solo se puede emplear como en poesía. Al poeta le interesa - dice él- no tanto la descripción de hechos cuanto la creación de imágenes" (en Bronowski, 1979: 340). También Aldoux Huxley afirmaba que "las ciencias de la vida necesitan las intuiciones del artista" (en Vilar, 1997: 242).

Recordemos, igualmente, que para la mente griega la belleza tuvo siempre una significación enteramente objetiva. La belleza era verdad, constituía un carácter fundamental de la realidad. De ahí nació el famoso lema, tan significativo y usado a lo largo de la historia del pensamiento filosófico: "lo verdadero, lo bueno y lo bello convergen"; es decir, "convergencia de la ciencia, la ética y el arte", pues solo la convergencia de estos tres aspectos del ser (lo que la fenomenología llama sus "esferas eidéticas" o "regiones del ser") nos daría la plenitud de la significación, la plenitud de "la verdad".

Como podremos observar, esta "plenitud de significación y de verdad" que nos daría la integración de estas tres "regiones del ser", equivale a lo que solemos considerar como un auténtico resultado de una sólida y rica formación personal y profesional, la cual nos lleva a la verdadera sabiduría, a la prudencia o sindéresis (como capacidad para juzgar rectamente). Esta sabiduría vendría a ser como una realidad emergente vivencial en la mente y vida del sujeto humano, que no se daría en los componentes que la constituyen, sino en su interacción recíproca. No es, por lo tanto, una disciplina tradicional, sino una metadisciplina, una transdisciplina. Esta "sabiduría" integraría los aspectos "verdaderos" de la realidad (ciencia) con su armonía y elegancia estética (arte) y con el respeto, aprecio y promoción de la naturaleza de esa realidad (ética). Esta tríada de saberes integrados es lo que la Unesco trata de señalar como el objetivo fundamental de toda renovación y replanificación universitaria.

Hoy día, después de la ilusión del pasado que nos hizo creer que la ciencia nos conduciría a un futuro de progreso infinito, y después de la amarga experiencia de Hiroshima, Nagasaki y Chernóbil, sabemos que la ciencia es ambivalente y que ya dispone del arsenal nuclear suficiente para convertir el planeta entero en un montón 
de cenizas y tan estéril como los demás planetas de nuestro sistema solar. Por ello, cobra suma importancia el papel de los estudios de sustentabilidad del medio ambiente y del papel que juega la ética.

Bertrand Russell, considerado uno de los pensadores más lúcidos del siglo XX y, quizá, de toda la historia de la humanidad, dice que "la ciencia, como persecución de la verdad, será igual pero no superior al arte" (1975: 8). Y Goethe señala que el "arte es la manifestación de las leyes secretas de la naturaleza" (en: Nietzsche, 1973: 127). Por esto, el mismo Gadamer concluye diciendo que "la oposición entre lo lógico y lo estético se vuelve dudosa" (1984: 656).

Estas mismas razones son las que han llevado a ilustres universidades como la de Harvard a pedir a sus estudiantes que el $25 \%$ de las asignaturas que cursen sean de áreas externas a su especialidad; e, igualmente, que en la Universidad Simón Bolívar (Caracas), desde su planificación, unos 40 créditos (15 cursos) sean de estudios generales, es decir, de formación personal, paralela a la formación profesional.

\section{CONCLUSIÓN}

Como consecuencia de todo lo dicho se deriva la importancia que tiene el clarificar e ilustrar que el problema en la mayoría de las controversias académicas reside en el concepto restrictivo de "cientificidad" adoptado, especialmente en las ciencias humanas, que mutila la legitimidad y derecho a existir de una gran riqueza de la dotación más típicamente humana, como los procesos que se asientan en el uso de la libertad y de la creatividad. Esta gran riqueza de dotación exige en el investigador, por un lado, una gran sensibilidad en cuanto concepto de las bases epistemológicas aceptadas y al uso de métodos, técnicas, estrategias y procedimientos para poder captarla y, por el otro, un gran rigor, sistematicidad y criticidad, como criterios básicos de la cientificidad requerida por los niveles académicos, criterios que, desde Kant para acá, constituyen su definición.

La unión de estos dos procesos investigativos ha exigido el desplazamiento de su ubicación, en el continuo ciencia-arte, desde la posición de una rigidez inadecuada para las ciencias humanas hacia una más cercana al arte; ha exigido un nuevo espacio bajo el concepto de "ciencia y arte"; es más, en varios de nuestros países, los ministerios de ciencia y tecnología ya han creado departamentos de "ciencia y arte", antes inconcebibles. Este espacio lo han ido tratando de ocupar, a lo largo de la segunda parte del siglo XX, las metodologías sistémico-cualitativas (cada una en su propio campo y a su manera) que, especialmente en este lapso, se han ido caracterizando por su esfuerzo en poseer las cualidades señaladas, indispensables para lograr conocimientos defendibles epistemológica y metodológicamente ante la comunidad científica internacional.

Pero, como dice Hegel (1966), “debemos estar convencidos de que lo verdadero tiene por naturaleza el abrirse paso al llegar su tiempo y de que solo aparece cuando este llega, razón por la cual nunca se presenta prematuramente ni se encuentra con un público aún no preparado para ello" (p. 47). 


\section{REFERENCIAS BIBLIOGRÁFICAS}

1. Aracil, J. (1986). Máquinas, sistemas y modelos. Madrid: Tecnos.

2. Aristóteles (1973). Obras completas. Madrid: Aguilar.

3. Bertalanffy, L. Von (1981). Historia y situación de la teoría general de sistemas. En Bertalanffy, L. Von y otros, Tendencias en la teoría general de sistemas. Madrid: Alianza.

4. Beynam (1978). The emergent paradigm in science. En ReVision Journal, 1, 2.

5. Bronowski, J. (1978). El sentido común de la ciencia. Barcelona: Pinínsula.

6. Bronowski, J. (1979). El ascenso del hombre. Caracas: Fondo educativo interamericano.

7. Capra, F. (1985). El punto crucial. Barcelona: Integral.

8. Capra, F. (1992). El tao de la física. Madrid: Luis Cárcamo.

9. Capra, F. (2003). La trama de la vida: una nueva perspectiva de los sistemas vivos. Barcelona: Anagrama.

10. CIRET (1994). Proceedings of world congress of transdisciplinarity: Centre international de recherches et etudes transdisciplinaires (CIRET). Proceedings from 1994 meeting in Lisbon (Portugal). Extraído de http://perso.club-/internet.fr/nicol/ciret/.

11. CIRET-UNESCO (1997). ¿Qué universidad para el mañana? Hacia una evolución transdisciplinaria de la universidad (declaración y recomendaciones). Congreso internacional sobre transdisciplinariedad, Locarno, Suiza, mayo de 1997.

12. CIRET-UNESCO (2000) International transdisciplinary conference, Zurich, 27 febrero-1 de marzo, 2000.

13. Clark, R. (1972). Einstein: the life and times. Nueva York: Avon Books.

14. Descartes, R. (1983). Discurso del método y reglas para la dirección de la mente. Barcelona: Orbis.

15. Dilthey, W. (1976). The rise of hermeneutics. En Connerton, P. (Dir.), Critical sociology. Nueva York: Penguin.

16. Eccles, J. C. y Popper, K. (1985). El yo y su cerebro. Barcelona: Labor.

17. Echeverría, J. (1989). Introducción a la metodología de la ciencia: la filosofía de la ciencia en el siglo XX. Barcelona: Barçanova.

18. Eco, U. (1990). La definición del arte: lo que hoy llamamos arte ¿ha sido y será siempre arte? Barcelona: Martínez Roca.

19. Eichner, A. (1989). Por qué la economía no es todavía una ciencia. En Rev. del Banco Central de Venezuela, julio-sept.

20. Feyerabend, P. (1975). Contra el método: esquema de una teoría anarquista del conocimiento. Barcelona: Ariel. 
21. Frey, G. (1972). La matematización de nuestro universo. Madrid: G. del Toro.

22. Gadamer, H. G. (1984). Verdad y método: fundamentos de una hermenéutica filosófica. Salamanca: Sígueme.

23. Gadamer, H. G. (1976). Philosophic hermeneutics. Berkeley: University of California Press.

24. Habermas, J. (1982). Conocimiento e interés. Madrid: Taurus.

25. Habermas, J. (1996). La lógica de las ciencias sociales. Madrid: Tecnos.

26. Habermas, J. (1999). Teoría de la acción comunicativa. Madrid: Taurus.

27. Hegel, G. (1966). Fenomenología del espíritu. México: FCE.

28. Heidegger, M. (1974). El ser y el tiempo. México: FCE.

29. Heisenberg, W. (1958a). Physics and philosophy: the revolution of modern science. Nueva York: Harper \& Row.

30. Heisenberg, W. (1958). The representation of nature in contemporary physics. En Daedalus, 87, 95-108.

31. Hertz, H. (1956). The principles of mechanics, presented in a new form. Nueva York: Dover.

32. Hospers, J. (1979). Philosphy of art. En Encyclopaedia Britannica. Inglaterra: Edic.

33. Kant, I. (1973). Crítica de la razón pura. Buenos Aires: Losada.

34. Kuhn, T. S. (1978). La estructura de las revoluciones científicas. México: F.C.E.

35. Lighthill, J. (1986). The recently recognized failure of predictability in newtonian dynamics. En Proceedings of the royal society, (tomo A, 35-50).

36. Mardones, J. M. (1991). Filosofía de las ciencias humanas y sociales: materiales para una fundamentación científica. Barcelona: Anthropos.

37. Martínez, M. (1986). La capacidad creadora y sus implicaciones para la metodología de la investigación. En Psicología (Caracas: UCV), XII, 1-2, 37-62.

38. Martínez, M. (1988). Enfoque sistémico y metodología de la investigación. En Anthropos, 16, 43-56.

39. Martínez, M. (1991). La inercia mental en los estudios de postgrado. En Argos, 14, 63-71.

40. Martínez, M. (1992). Significación de la matriz epistémica en los estudios de postgrado. En Anthropos, 24, 5-14.

41. Martínez, M. (1993). El proceso creador a la luz de la neurociencia. En Comportamiento, 2, 1, 3-22.

42. Martínez, M. (1996). Comportamiento humano: nuevos métodos de investigación. México: Trillas. 
43. Martínez, M. (1997). La matematización del saber y sus límites: mito y realidad de los modelos matemáticos. En Argos, 25, 103-130.

44. Martínez, M. (1997). El paradigma emergente: hacia una nueva teoría de la racionalidad científica. México: Trillas

45. Martínez, M. (1998). La investigación cualitativa etnográfica en educación: manual teórico-práctico. México: Trillas.

46. Martínez, M. (1999). La nueva ciencia: su desafío, lógica y método. México: Trillas.

47. Martínez, M. (1999). La psicología humanista: un nuevo paradigma psicológico. México: Trillas.

48. Martínez, M. (2000). El proceso de nuestro conocer postula un nuevo paradigma epistémico. En Rev. RELEA, 11, 15-36.

49. Martínez, M. (2001). Necesidad de un nuevo paradigma epistémico. En Las ciencias sociales: reflexiones de fin de siglo. Caracas: Edit. Trópykos, Univ. Central de Venezuela.

50. Martínez, M. (2001). Uso del programa computacional Atlas.ti en la estructuración de "datos" cualitativos. En Argos, 34, 139-156.

51. Martínez, M. (2003). Transdisciplinariedad y lógica dialéctica: un enfoque para la complejidad del mundo actual. En Conciencia activa, 21, 1, 107-146.

52. Martínez, M. (2004). Ciencia y arte en la metodología cualitativa. México: Trillas.

53. Martínez, M. (2007). Evaluación cualitativa de programas. México: Trillas.

54. Martínez, M. (2008). Epistemología y metodología cualitativa en las ciencias sociales. México: Trillas.

55. Martínez, M. (2009). Nuevos paradigmas en la investigación. Caracas: Alfa.

56. Merleau-Ponty, M. (1975). Fenomenología de la percepción. Madrid: Península.

57. Merleau-Ponty, M. (1976). La estructura del comportamiento. Buenos Aires: Hachette.

58. Morin, E. (1982). Para salir del siglo XX. Barcelona: Kairós.

59. Morin, E. (1983). El método. Vol. II: la vida de la vida. Madrid: Cátedra.

60. Morin, E. (1984). Ciencia con conciencia. Barcelona: Anthropos.

61. Nietzsche, F. (1972). Más allá del bien y del mal. Madrid: Alianza.

62. Nietzsche, F. (1973). En torno a la voluntad de poder. Barcelona: Península.

63. Pareyson, L. (1992). Estetica-Teoria della formatività. Bolonia.

64. Piaget, J. (1975). Psicología y epistemología. Madrid: Ariel. 
65. Polanyi, M. (1958). Personal knowledge. Chicago: University of Chicago Press.

66. Polanyi, M. (1966). El estudio del hombre. Buenos Aires: Paidós.

67. Polanyi, M. (1968). Science in a free society. Londres: NBL.

68. Polanyi, M. (1969). Knowing and being. Londres: Routledge and Kegan Paul.

69. Popper, K. (1973). La lógica de la investigación científica. Madrid: Tecnos.

70. Popper, K. (1977). Búsqueda sin término: una autobiografía intelectual. Madrid: Tecnos.

71. Popper, K. y Eccles, J. (1985). El yo y su cerebro. Barcelona: Labor Universitaria.

72. Racionero, L. y Medina, L. (1990). El nuevo paradigma. Barcelona: Promociones y Publicaciones Universitarias.

73. Ricoeur, P. (1969). Le conflit des interprétations. París: Seuil.

74. Rivadulla, A. (1986). Filosofía actual de la ciencia. Madrid: Tecnos.

75. Rogers, C. R. (1980). A way of being: the latest thinking on a person-centered approach to life. Boston: Houghton.

76. Rogers, C. R. (1980). El poder de la persona. México: El Manual Moderno.

77. Russell, B. (1975). La perspectiva científica. Barcelona: Ariel.

78. Saussure, F. de (1954). Curso de lingüística general. Buenos Aires: Losada.

79. Schrödinger, E. (1967). What is the life? \& mind and mater. Inglaterra: Cambridge Univ. Press.

80. Sheldrake, R. (1990). Una nueva ciencia de la vida. Barcelona: Kairós.

81. Suppe, F. (1979). La estructura de las teorías científicas. Simposio: Urbana, IL., EE.UU., marzo de 1969). Madrid: Editora Nacional.

82. Tarski, A. (1956). Logic, semantics and metamathematics. Oxford: Clarendon Press.

83. UNESCO (1998). Transdisciplinarity: towards integrative process and integrated knowledge. Simposio en Royaumont (Francia). Tomado de http://firewall, unesco. org/philosophy/transdisciplinarity.

84. Union of International Associations (1994). Transdisciplinarity through structured dialogue. Bruselas, Bélgica. Tomado de http://www.uia.org.

85. Vilar, S. (1997). La nueva racionalidad: comprender la complejidad con métodos transdisciplinarios. Barcelona: Kairós.

86. Weimer, W. B. (1979). Notes on the methodology of scientific research. Nueva Jersey: Wiley.

87. Wittgenstein, L. (1969). Philosophical investigations. Nueva York: Macmillan.

88. Wittgenstein, L. (1973). Tractatus logico-philosophicus (versión bilingüe alemáncastellano). Madrid: Alianza. 